\title{
BOTSWANA-BAYLOR CHILDREN'S CLINICAL CENTRE OF EXCELLENCE
}

\section{The role of a paediatric centre of excellence in assuring the prioritisation and quality of paediatric care within a developing national HIV care programme}

\author{
Elizabeth D Lowenthal, $M D^{\prime}$ \\ Gabriel M Anabwani, $M B C h B, M M e d, M S c^{l}$ \\ Haruna B Jibril, $M B B S$, PMCPaed, $M S c^{2}$ \\ Beth Barr, $M P H^{1}$ \\ Opelo M Rankopo, $R N, B N, M N^{t}$ \\ Mark W Kline, $M D^{1}$ \\ ${ }^{1}$ Baylor College of Medicine, Baylor International Pediatric AIDS Initiative, \\ Botswana-Baylor Children's Clinical Centre of Excellence \\ ${ }^{2}$ Princess Marina Hospital, Botswana
}

\begin{abstract}
The HIVIAIDS epidemic is having a devastating global impact on children and families. In 2004, an estimated 640000 children became newly infected with HIV and about 2.2 million children were living with the virus. More than half a million children worldwide died of HIV-related illnesses in 2004. Ninety-five per cent of HIVIAIDS-associated deaths occur in the developing world and about 70\% of infected individuals reside in subSaharan Africa. 'Worldwide, HIV is threatening child survival and reversing development gains of past decades. Globally, HIV/AIDS now accounts for about $4 \%$ of all deaths among children under 5 years of age; in southern Africa, HIVIAIDS may account for 30 - 50\% of all such deaths. Where paediatric HIVrelated care and treatment services are not available, it is estimated that more than half of infected children die before reaching their second birthday. ${ }^{2}$
\end{abstract}

When comprehensive care, including highly active antiretroviral therapy (HAART), is available to children, these grim statistics can be reversed. Survival rates are significantly better among children and adolescents treated with HAART.-5 Growth is normalised ${ }^{6,7}$ and children thrive in normal school environments. ${ }^{8}$ In a resource-poor setting it has been demonstrated that HAART can be administered safely and effectively. ${ }^{6,9}$

Despite the great potential for children to thrive within childfocused treatment programmes in both high- and low-resource settings, 'children are for the most part ignored"10 within international HIV resource planning initiatives. When their care is included in programme planning, children are most commonly 'treated as an "add on" to the adult response.'10 Children represent a disproportionate share of those individuals infected with HIV who do not have access to treatment. ${ }^{11,12}$

Contributing to the limited availability of paediatric care is the lack of health professionals with expertise or experience in treating children infected with HIV, including the provision of therapy, dosing, administration and monitoring. Worldwide, health care providers at all levels have limited skills to identify children living with HIV; to provide them with antiretroviral (ARV) treatment and other care services; to monitor their progress; and to provide psychosocial support. Most health care professionals in countries hard-hit by HIV lack formal training in paediatric HIV/AIDS treatment. Formal research training and infrastructure for the performance of paediatric-focused HIVIAIDS clinical research are also lacking. The creation of paediatric clinical centres of excellence facilitates the prioritisation of paediatric HIV care services within and around countries hard-hit by the HIV epidemic and assures that highquality paediatric-focused health care education is available.

\section{THE BOTSWANA EXPERIENCE}

In Botswana, nearly $40 \%$ of all pregnant women are infected with HIV. ${ }^{13}$ Standard surveillance does not include children, so exact paediatric numbers are unknown. It is thought, however, that thousands of Batswana children under the age of 15 are HIV-infected. Because of a strong nationwide commitment, led by the President of the Republic of Botswana, His Excellency Mr Festus Mogae, Botswana became the first country in subSaharan Africa to establish a national ARV treatment programme. In 2000, the government of Botswana entered the African Comprehensive HIV/AIDS Partnership (ACHAP), ${ }^{14}$ in order to establish a sustainable response to the country's epidemic. In 2001, the government of Botswana made an unprecedented commitment to provide free HAART to all qualifying citizens through its national ARV programme.

For paediatric patients, ARV medications became available through the public sector in Botswana in May 2002. In the year before that, children in need of ARV therapy were able to obtain necessary medications through a clinical trial managed jointly by staff of the Princess Marina Hospital and Baylor College of 
Medicine. Theoretical and practical training was also performed jointly to prepare available staff to lead the nation in the provision of excellent care and treatment to HIV-infected children. An exchange programme was established to allow Botswana-based faculty to obtain further practical training in research and patient care in the USA and to allow US-based health professionals to work in the clinic in Botswana.

In 2001, an adult Infectious Disease Care Clinic was established as a pilot at Princess Marina Hospital. This clinic formed the backbone of the National Antiretroviral Programme for adults beginning in January 2002. Space for the care of paediatric patients was available at the site for half a day each week, beginning in June 2002. To better address the unique needs of the paediatric population, a centre dedicated to providing comprehensive, state-of-the-art care and treatment to HIVinfected children was established on the Princess Marina Hospital campus. The Botswana-Baylor Children's Clinical Centre of Excellence (COE) was opened and officially dedicated by His Excellency Mr Festus Mogae on 20 June 2003. This facility, the first of its kind on the African continent, provides state-of-the-art care and treatment to HIV-infected children and families from Gaborone and across Botswana. The Centre is a product of a partnership between the Baylor International Pediatric AIDS Initiative at Baylor College of Medicine (Houston, Texas, USA), the Princess Marina Hospital in Gaborone and the government of Botswana. The centre was made possible by a landmark \$6 million grant from the Bristol-Myers Squibb Secure the Future Programme. The centre is supported by additional funding to Baylor from the Fogarty International Center of the US National Institutes of Health and the US Centers for Disease Control and Prevention Global AIDS Program. The COE is based on a model first piloted in Constanta, Romania, another resource-poor setting with a significant paediatric HIV epidemic., ${ }^{6,9}$

Staffed collaboratively by US and Botswana health professionals, the Botswana-Baylor Children's Clinical Centre of Excellence (COE) provides services that are comprehensive in scope, encompassing the primary and specialty medical care and social service needs of HIV-infected infants, children and families. The centre includes ten outpatient clinic rooms, a pharmacy, a laboratory, a classroom, offices and a conference room. Health professional education and clinical research are integral to the centre's mission. By prioritising health professional educational activities, the centre catalyses the decentralisation and expansion of paediatric care throughout Botswana and the region.

\section{PATIENT CARE}

The heart of all initiatives organised through the COE is the provision of outstanding care and treatment to HIV-infected children. Paediatric care in Gaborone had been initiated at the Princess Marina Hospital about a year before and was transferred to the COE at the time of its opening in June 2003. Even as the national programme services expanded to include children throughout the country, the COE remained the focal point of paediatric care nationwide

Currently, the majority of patients receiving care through the COE are enrolled in the Botswana National Antiretroviral Programme. At the $\mathrm{COE}$, the National Paediatric Antiretroviral Programme is jointly administered by the paediatric staff of the Princess Marina Hospital and the COE. Children enrolled in the programme at the $\mathrm{COE}$ receive comprehensive care including primary paediatric care, antiretroviral therapy and monitoring, psychosocial support and nutritional surveillance from a dedicated and multidisciplinary staff.

A Family Care Model clinic has also been established to provide care to caretakers along with their children. At the COE, paediatricians are available to care for children and adult patients receive treatment from a physician specialist. In nonspecialist centres throughout the country, it is anticipated that families will similarly receive their care together using the family care model. As the availability of comprehensive care for HIV-infected children extends to the rest of the nation, most children and families will be treated by more generally trained medical officers. The Botswana Baylor Children's Clinical Centre of Excellence will remain as a referral centre for the most challenging paediatric cases as well as for ongoing training of health care providers in optimal treatment strategies for children.

\section{EDUCATION}

As is true for most of sub-Saharan Africa, Botswana has a very limited number of paediatric specialists. The majority of all specialists work at the two national referral hospitals in Francistown and Gaborone. General practitioners in the national ARV programme are frequently hesitant to screen and treat children for HIV. In order to address this issue, the COEbased staff from both the Baylor Programme and Princess Marina Hospital conduct week-long training courses at the 'ARV roll-out sites' around Botswana. These trainings focus on issues related to paediatric HIV care and antiretroviral treatment and are both didactic and practical. These educational programmes are funded by the US Centers for Disease Control and Prevention (CDC) Botswana-USA (BOTUSA) project. The COE also collaborates with the Elizabeth Glazer Pediatric AIDS Foundation (EGPAF) to provide training for a number of health professionals from countries throughout the region. The training serves to strengthen the capacity to provide ARVs to children throughout the country and the region. Following the formal training, practitioners from the remote sites frequently consult with the COE-based specialists for guidance with their difficult paediatric cases.

Trainees attend clinic and didactic sessions at the COE, or at their home sites with COE-based visiting preceptors, in order to increase their skills in treating paediatric HIV. African countries benefiting from these training activities have included South Africa, Swaziland, Zambia, Nigeria and Tanzania. The topics 
covered in the paediatric training programmes include those shown in Fig. 1.

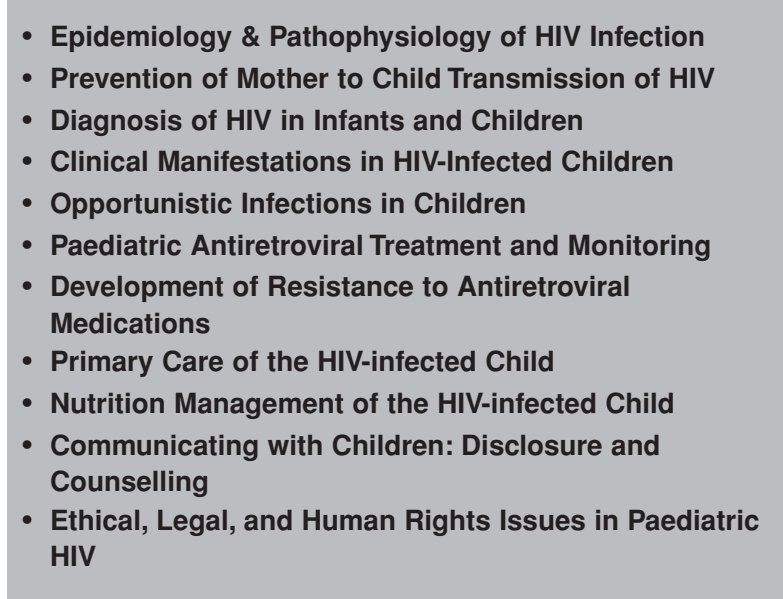

Fig. 1. Paediatric HIV training modules.

Training is inclusive of a number of health professional groups including students, nurses, doctors and pharmacists. A curriculum on paediatric HIVIAIDS for health professionals has also been developed to allow for the wider dissemination of practical knowledge. ${ }^{15}$ This teaching tool is available in several languages and has been disseminated to health care workers in more than 50 countries.

\section{RESEARCH}

The long-term provision of care and treatment to HIV-infected children worldwide will be limited by the relative lack of data regarding the safest and most effective employable options for children. ${ }^{16}$ In order to address the knowledge gap regarding the unique needs of children infected with HIV, the COE provides infrastructure, expertise and staffing to conduct high-quality, high-impact, highly ethical paediatric clinical research.

Currently enrolment is underway for the Botswana/Baylor Antiretroviral Assessment Trial 2 (BANA02), an analysis of CD4positive T-lymphocyte count-based structured treatment interruptions in children. With targeted enrollment of 600 children, BANA 2 will be the largest paediatric ARV clinical trial to have been performed on the African continent. (BANA means children in Setswana. BANA 2 is the second large study dedicated to expanding the knowledge related to ARV therapy options for children in Botswana.)

Research organised through the COE also addresses barriers to paediatric ARV adherence, disclosure of HIV status to children and adolescents, and the comprehensive needs of high-risk paediatric populations.

\section{OUTREACH}

In order to facilitate the extension of high-quality paediatric care to the broader community, the COE has initiated a number of outreach programmes. These programmes share a common focus on improving the access to high-quality medical care for children, including orphans and vulnerable children (OVCs). The methods employed through outreach programmes mirror others organised through the COE, stressing the provision of high-quality medical care, psychosocial support and training.

The COE staff, supported by a separate grant from Secure the Future, also provide on-site support to health care professionals in Bobonong, Botswana. With over $70 \%$ of women of childbearing age testing positive for HIV in the Bobirwa subdistrict, the people of this area suffer from one of the highest prevalence rates of HIV infection of any community in the world. Before the COE's involvement there, no patients were receiving ARV treatment through this site. Because of the outreach work at this site, patients are being started on therapy more rapidly and the prioritisation of paediatric care is consistently stressed.

Closer to the $\mathrm{COE}$, another outreach programme is working in collaboration with community-based organisations (CBOs) to perform health assessments of more than a thousand OVCS living in three densely populated villages within $50 \mathrm{~km}$ of Gaborone. The COE is taking the lead in Botswana in the area of home-based testing. Children connected to orphan programmes in the community are visited in their homes, and all family members are counselled and offered age-appropriate HIV testing. Adults and children over 18 months of age can receive immediate at-home rapid-testing. Children who are found to be HIV-infected or to have other special needs are then appropriately referred, many directly to the COE for ARV treatment.

\section{OUTCOMES}

As of 1 April 2005, 3937 Batswana children had been screened for HIV infection at the Botswana Baylor Children's Clinical Centre of Excellence. Of these, 1353 children (34.4\%) were HIVpositive, and 1159 paediatric patients have begun HAART at the COE. Only 61 patients are known to have died since testing positive for HIV at the COE. Most of these deaths occurred within a short time of the children's coming to the COE, in patients who presented with advanced disease. Twenty of the children died before they could be started on ARV medications.

The first 214 children to complete 12 months of follow-up within the COE-based national programme have been systematically evaluated with respect to medication adherence, clinical condition, CD4\% and viral load values. At the time of entry into the programme, 121 (56.5\%) were in CDC category C3, indicating a severe clinical state. Owing to the exceptional commitment of the patients' families, complemented by intensive psychosocial support, overall medication adherence (based on pill count and liquid medication measurements) was 95\% during the initial year of follow-up. Of the patients $85 \%$ achieved complete virological suppression (viral load $<400$ ). Mean log viral load values at entry, 3 months, 6 months, and 12 months of follow-up are demonstrated in Fig. 2. Fig. 3 


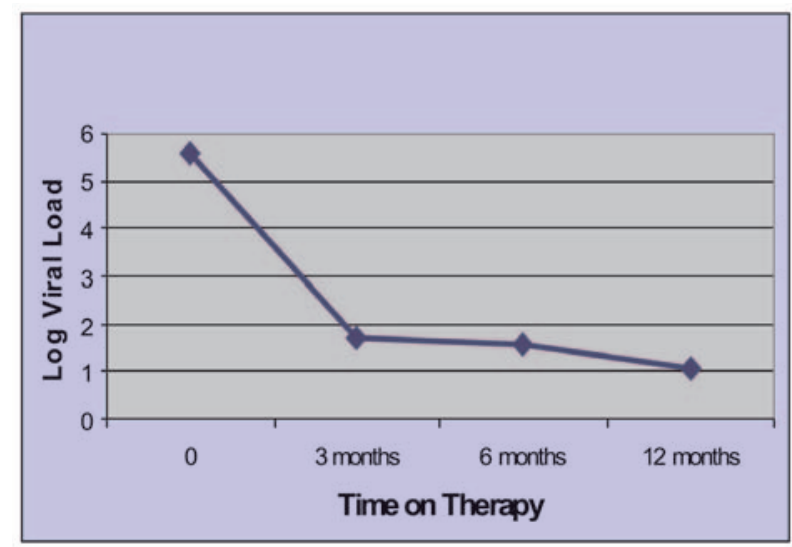

Fig. 2. Mean log viral load during first year of therapy.

illustrates the patients' mean CD4\% during the same time period. An excellent clinical, immunological and virological response to ARV therapy was achieved.

\section{DISCUSSION}

The creation of dedicated paediatric centres of excellence is transforming the face of paediatric HIV/AIDS, providing treatment for the first time to large numbers of infected children, building essential capacity and infrastructure, and promoting the acquisition of knowledge that will enhance HIV/AIDS care and treatment globally. We credit the early success of the Botswana-Baylor Children's Clinical Centre of Excellence to a variety of factors: a commitment to comprehensive medical, psychosocial, and community support; well-trained multidisciplinary staff; dedicated collaborative partners; highly motivated children and caregivers; and attention to family-centred care.

The Botswana government's commitment to sustainability of the national ARV programme and to the treatment of children has ensured that our efforts to prioritise paediatric care have received consistent support. The Baylor College of Medicine's early partnership with the Princess Marina Hospital has allowed for ongoing sharing of resources, essential to the daily function of the COE. The necessity for treatments that focus on children has been recognised and honoured by all COE partners, leading to consistent prioritisation and a high quality of care for a growing number of Botswana's children. Currently, additional Paediatric Clinical Centres of Excellence for the care and treatment of HIV-infected children are being built based on the success of Botswana's programme. Centres in Swaziland and Lesotho, also funded by the Bristol Myers Squibb Secure the Future Programme, are scheduled to open in December of 2005.

\section{REFERENCES}

1. UNAIDS. 2004 Report on the global AIDS epidemic. http://www. unaids.org/wad2004/report.html (accessed 2 April 2004).

2. Newell ML, Coovadia H, Cortina-Borjam, et al. Mortality of infected and uninfected infants born to HIV-infected mothers in Africa: a pooled analysis. Lancet 2004; 364: 1236-1243.

3. McConnell MS, Byers RH, Frederick T, et al. Trends in antiretroviral use and survival rates for a large cohort of HIV-infected children and adolescents in the United States, 1989-2001. JAIDS 2005; 389(4): 488494.

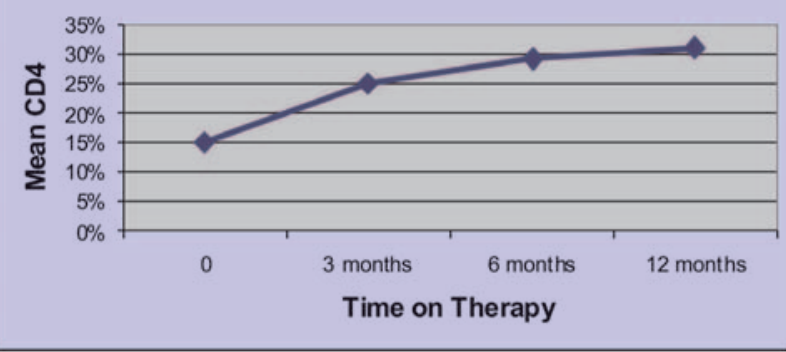

Fig. 3. Mean CD4\% during first year of therapy.

4. Gortmaker SL, Hughes M, Cervia J, et al. Effect of combination therapy including protease inhibitors on mortality among children and adolescents infected with HIV-1. N Engl J Med 2001; 345: 1522-1528.

5. de Martino $M$, Tovo $P$, Balducci $P$, et al. Reduction in mortality with availability of antiretroviral therapy for children with perinatal HIV-1 infection. JAMA 2000; 284: 190-197.

6. Kline MW, Matusa RF, Copaciu L, et al. Comprehensive pediatric human immunodeficiency virus care and treatment in Constanta, Romania. Implementation of a program of highly active antiretroviral therapy in a resource-poor setting. Pediatr Infect Dis J 2004; 23: 695-700.

7. Verweel G, van Rossum AM, Hartwig NG, Wolfs TF, Scherpbier HJ, Groot R. Treatment with highly active antiretroviral therapy in human immunodeficiency virus type 1-infected children is associated with a sustained effect on growth. Pediatrics 2002; 109: e25.

8. Wilfert $\mathrm{CM}$, Kline MW, Futterman D, et al. Education of children with human immunodeficiency virus infection. Pediatrics 2000; 105: 13581360.

9. Kline MW. No greater gift than hope. Seminars in Pediatric Infectious Diseases 2003; 14: 309-313.

10. UNICEF. Reaching out to children in the WHO ' 3 by 5 ' initiative: paediatric HIV care and treatment summary report.

11. UNICEF/WHO Technical Consultation. Improving access to appropriate paediatric ARV formulations. 3 - 4 November 2004. http://www.who. int/3by5/en/finalreport.pdf (accessed 2 April 2005).

12. United Nations Children's Fund. The State of the World's Children 2005. UNICEF, 2004.

13. Botswana National AIDS Coordinating Agency. 2003 Second Generation HIV/AIDS Surveillance Technical Report. Gaborone: Botswana National AIDS Coordinating Agency, 2003.

14. Ramiah I, Reich MR. Public-private partnerships and antiretroviral drugs for HIV/AIDS: Lessons from Botswana. Health Affairs 2005; 24: 545-551.

15. Baylor International Pediatric AIDS Initiative. HIV Curriculum for the Health Professional. Houston: Baylor College of Medicine, 2003.

16. World Health Organization. HIV medicines. 3 by 5 strategy: improving access to paediatric HIV medicines. WHO Drug Information 2004; 18: 288-

The work at the Botswana Baylor Children's Centre of Excellence would never have been realised without the support of the Botswana Ministry of Health, Princess Marina Hospital, Baylor College of Medicine and the Bristol Myers Squibb Secure the Future Programme. We also wish to acknowledge the contributions of CDC-BOTUSA, the Elizabeth Glaser Pediatric AIDS Foundation, the Fogarty International Center of the US National Institutes of Health, the Kgalagadi Beverages Trust and Debswana. The ongoing dedicated work of the staff of the COE and Princess Marina Hospital, our patients, and their families, ensure the continued success of these programmes. 